diary filled out by the nurses at the end of each work-shift provided information concerning family and social relationships during a typical working week. Habits on coffee, smoke, time of meal assumption and home management during working days were also collected.

Result Nurses working on CCW shift rotation reported more frequently difficulties in keeping adequate family and social relationships compared to nurses working on CW one $(96 \%$ vs $73 \%, \mathrm{p}=0.002$ ).

No differences were reported in coffee (3-4 cups/day), smoking (61\%) habits and time of meals assumption (irregular in about $33 \%$ of nurses) during working days in the two groups. No differences were reported in the time spent in home management by the two groups.

Discussion CCW shift rotation seemed to disrupt quality of family and social relationships of nurses more than $\mathrm{CW}$ one. The fact that CCW shift rotation is associated to higher sleep disturbances and more fatigue in the free-time might partially explain these results. These aspects should be taken into account in shift-work schedule organisation, particularly in women.

\section{SHIFTING TIMES: RECENT ROTATIONAL SHIFTWORK AND INCIDENT HYPERTENSION RISK}

1J Ferguson*, 'S Costello, ${ }^{2} \mathrm{M}$ Cullen, ${ }^{1} \mathrm{E}$ Eisen. ${ }^{1}$ School of Public Health, University of California Berkeley, Berkeley, California, USA; ${ }^{2}$ Stanford University, Stanford, California, USA

\subsection{6/oemed-2018-ICOHabstracts.1369}

Introduction Rotational shiftwork, such as alternating day, afternoon, and night shifts, causes chronobiologic phase disruptions which may cause an increase in hypertension risk. Prior research has classified rotational shiftwork using assigned schedules in lieu of actual recorded work which may result in exposure misclassification. Therefore, we assessed the association between a novel quantitative definition of rotational shiftwork and incident hypertension.

Methods A cohort of 2156 new hires at 9 aluminium smelter and fabrication facilities were followed from 2003 through 2013 for incident hypertension defined by ICD-9 codes from insurance claims. Detailed time-registry data was used to classify each worker's history of rotational shiftwork. Rotational shiftwork was defined as shift with a larger than 4 hour absolute value change in start time from the preceding shift that started on an earlier day. The association between incident hypertension and rotational shiftwork in the previous 12 months was estimated in a Cox proportional hazards model, adjusting for age, sex, facility, smoking history, annual BMI, annual health claims based risk score, night shift exposure (percentage of shifts that were night shifts cumulatively and in the previous year), and history of rotational work.

Results The majority of workers (99.8\%) experienced at least one rotation in their work history. The average worker had at 2.91 rotations $(\mathrm{SD}=1.87)$ per month with a mean of 130 rotations over their work history. Compared with workers with less than $5 \%$ rotational shiftwork in the previous year, the hazard ratio among workers with $\geq 5-<15 \%, \geq 15-<30 \%$, and $\geq 30 \%$ rotational shiftwork in the previous year were 1.02 $(0.64-1.64), 1.13(0.73-1.76)$, and 1.53 (0.95-2.45) respectively. Estimates were robust to the adjustment for night shifts Conclusion Our results suggest recent rotational shiftwork exposure may be associated with higher rates of hypertension.
Furthermore, rotational shiftwork may have an independent effect on hypertension risk not explained by night work.

\section{INFLUENCE OF CHRONOTYPE AND SHIFTWORK ON SLEEP DURATION AND OCCUPATIONAL ACCIDENTS: FINDINGS FROM A CROSS-SECTIONAL STUDY IN METAL WORKING INDUSTRY}

${ }^{1}$ Barbara Hirschwald*, ${ }^{1}$ Yi Sun, ${ }^{2}$ Thomas Heitmann, ${ }^{1}$ Frank Bochmann. ${ }^{1}$ Institute for Occupational Safety and Health of the German Social Accident Insurance, St. Augustin, Germany; ${ }^{2}$ German Social Accident Insurance Institution for the woodworking and metalworking industries, Germany

\subsection{6/oemed-2018-ICOHabstracts. 1370}

Introduction Atypical work-times such as night shifts and very early shifts can affect the quality and quantity of sleep. Short sleep duration and disturbed sleep are associated with a hightened accident risk. Humans and animals are influenced by a biological clock which is genetically determined and synchronised by daylight. The study investigates the impact of the start of working time and chronotype on sleep duration and occupational accidents.

Methods In this cross-sectional study data were collected from about 550 employees in the woodworking and metalworking industry. Analyses were conducted for relationships between chronotype, start of working time, sleep duration and accidents.

Result The average chronotype moves by two hours towards an earlier type from the youngest to the oldest group of employees. Among the subjects without accidents 23\% regard themselves as morning types, 69\% as intermediate types and $9 \%$ as evening types. Among the subjects who had an accident $32 \%$ were morning types and 5\% evening types. The late chronotypes get the least sleep on workdays and the early chronotypes get the least sleep on work-free days. Significant differences in the sleep duration were found for morning shift workers compared to day workers. A relevant proportion of the sleep deficit is due to the early start of working time.

Discussion The study shows significant chronotype-dependant differences in the sleep duration on work-days and free days. Early chronotypes seem to be unable to compensate their sleep deficit on free days, late types are unable to sleep early in the evening. Results will be useful to identify groups of workers at hightened risk for sleep deficit and sleepiness at work. In this way occupational accidents in shift workers and workers with an early starting time of work could be reduced.

\section{HEALTHY DIET AND REDUCTION OF CHRONIC DISEASE RISKS OF NIGHT SHIFT WORKERS - A REVIEW}

GM Ferri*, D Cavone, G Intranuovo, F Birtolo, E Lepore, L Macinagrossa. University of Bari, Italy, Interdisciplinary Department of Medicine (DIM), Section 'B. Ramazzini', Universitary Regional Hospital 'Policlinico - Giovanni XXIIIth', Unit of Occupational Medicine

\subsection{6/oemed-2018-ICOHabstracts. 1371}

Introduction The large increase of epidemiological studies on night shift work is due to the important effects on workers' health and psychophysical wellbeing. The short-term effects are easily studied and they are: insomnia, difficulties in managing work and private life, lower work performance, more 